University of Wollongong

Research Online

Faculty of Engineering and Information

Faculty of Engineering and Information

Sciences - Papers: Part B

Sciences

2018

On monolithic silicon array detectors for small-field photon beam dosimetry

Giordano Biasi

University of Wollongong, gb446@uowmail.edu.au

Jeremy A. Davis

University of Wollongong, jeremyd@uow.edu.au

Marco Petasecca

University of Wollongong, marcop@uow.edu.au

Susanna Guatelli

University of Wollongong, susanna@uow.edu.au

Vladimir Perevertaylo

SPA BIT

See next page for additional authors

Follow this and additional works at: https://ro.uow.edu.au/eispapers1

Part of the Engineering Commons, and the Science and Technology Studies Commons

Research Online is the open access institutional repository for the University of Wollongong. For further information contact the UOW Library: research-pubs@uow.edu.au 


\title{
On monolithic silicon array detectors for small-field photon beam dosimetry
}

\author{
Abstract \\ Contemporary $x$-ray radiotherapy employs small radiation fields to deliver highly conformal dose \\ distributions. Submillimeter accuracy in the measurement of the delivered dose map is a crucial \\ requirement of detectors proposed for quality assurance applications. 2D monolithic silicon array \\ detectors can provide high spatial-resolution by optimizing small sensitive volumes (SVs) in a large active \\ area. They offer a stable and near energy-independent response in megavoltage photon beams, good \\ dose linearity and real-time read-out. The SVs are ion-implanted on a silicon wafer whose geometry and \\ physical characteristics, such as resistivity and defects concentration, dramatically affect the detector \\ performance. The Octa is a novel 2D monolithic silicon array detector dedicated to small-field dosimetry. \\ Its 512 diode-SVs are arranged with a sub-millimeter pitch along 4 intersecting orthogonal linear arrays. \\ We report on the experimental and numerical characterization (performed with Sentaurus Workbench \\ within the Synopsys framework) of two Octa detectors, manufactured respectively on a bulk and on an \\ epitaxial silicon substrate. The effects of resistivity and defects concentration profiles across their large- \\ area monolithic silicon wafers is compared and discussed in terms of the response linearity with dose, \\ response uniformity, charge-collection efficiency and clinical performance in the case of a small radiation \\ field delivered with a flattening filter free beam. \\ Disciplines \\ Engineering | Science and Technology Studies \\ Publication Details \\ Biasi, G., Davis, J., Petasecca, M., Guatelli, S., Perevertaylo, V., Kron, T. \& Rosenfeld, A. B. (2018). On \\ monolithic silicon array detectors for small-field photon beam dosimetry. IEEE Transactions on Nuclear \\ Science, 65 (9), 2640-2649.

\section{Authors} \\ Giordano Biasi, Jeremy A. Davis, Marco Petasecca, Susanna Guatelli, Vladimir Perevertaylo, Tomas Kron, \\ and Anatoly B. Rosenfeld
}




\title{
On monolithic silicon array detectors for small-field photon beam dosimetry
}

\author{
Giordano Biasi, Jeremy Davis, Member, IEEE, Marco Petasecca, Member, IEEE, Susanna Guatelli, \\ Vladimir Perevertaylo, Tomas Kron and Anatoly B. Rosenfeld, Senior Member, IEEE
}

\begin{abstract}
Contemporary $\mathrm{x}$-ray radiotherapy employs small radiation fields to deliver highly conformal dose distributions. Sub-millimetre accuracy in the measurement of the delivered dose map is a crucial requirement of detectors proposed for quality assurance applications.

2D monolithic silicon array detectors can provide high spatialresolution by optimizing small sensitive volumes (SVs) in a large active area. They offer a stable and near energy-independent response in megavoltage photon beams, good dose linearity and real-time read-out. The SVs are ion-implanted on a silicon wafer whose geometry and physical characteristics, such as resistivity and defects concentration, dramatically affect the detector performance. The Octa is a novel $2 \mathrm{D}$ monolithic silicon array detector dedicated to small-field dosimetry. Its $\mathbf{5 1 2}$ diode-SVs are arranged with a sub-millimeter pitch along 4 intersecting orthogonal linear arrays.

We report on the experimental and numerical characterization (performed with Sentaurus $^{\mathrm{TM}}$ Workbench within the Synopsys ${ }^{\circledR}$ framework) of two Octa detectors, manufactured respectively on a bulk and on an epitaxial silicon substrate. The effects of resistivity and defects concentration profiles across their largearea monolithic silicon wafers is compared and discussed in terms of the response linearity with dose, response uniformity, charge-collection efficiency and clinical performance in the case of a small radiation field delivered with a flattening filter free beam.
\end{abstract}

Index Terms-2D monolithic silicon array detector, small-field dosimetry, flattening filter free beams, Sentaurus TCAD, chargecollection efficiency

\section{INTRODUCTION}

Contemporary x-ray radiotherapy employs small radiation fields $(<3 \mathrm{~cm}$ side) to deliver highly conformal

Manuscript received April 17, 2018; revised July 13, 2018; accepted July 25 , 2018. Date of current version July 25, 2018. We would like to acknowledge the Gross Foundation for financial support and ANFF Design House and Multi-modal Australian ScienceS Imaging and Visualization Environment (MASSIVE) for usage of Synopsys ${ }^{\circledR}$ TCAD. We thank M. A. Ebert, G. Grogan, B. H. Hug and J. Lane at the Sir Charles Gairdner Hospital for access to their CyberKnife ${ }^{\circledR}$.

G. Biasi, J. Davis, M. Petasecca, S. Guatelli, T. Kron and A. B. Rozenfeld are with the Centre for Medical Radiation Physics, University of Wollongong, NSW 2522, Australia (e-mail: gb446@uowmail.edu.au).

V. Perevertaylo is with the SPA-BIT, Kiev 02232, Ukraine.

T. Kron is with the Peter MacCallum Cancer Centre, Melbourne, VIC 3000 Australia and with the Sir Peter MacCallum Cancer Institute, University of Melbourne, VIC 3010, Australia.

Color versions of one or more of the figures in this paper are available online at http://ieeexplore.ieee.org.

Digital Object Identifier: dose distributions to the target. To avoid dosimetric inaccuracies in the quality assurance (QA) process, which may cause poor patients outcomes [1], [2], sub-millimetre spatial resolution in the measurement of the delivered dose map is a crucial detector requirement. Currently, the only commercially available options able to satisfy this requirement are point detectors used with various scanning techniques [2].

A preferable solution would be a suitable $2 \mathrm{D}$ detector. In particular, 2D monolithic silicon arrays can be fabricated of relatively large area while optimizing the spatial resolution with small sensitive volumes (SVs) [3]-[6]. They would offer advantages in terms of QA applications. Along with commonly characterized parameters, such as output factors (OFs), percentage depth dose (PDD) and tissue maximum ratio (TMR) distributions, and out off-axis ratios (OARs), their fixed geometry would allow for accurate reproducible machine-specific QA. Examples would include the positional accuracy verification of the movable parts of a medical linear accelerator (linac), such as the leaves of a multi-leaf collimator (MLC) and the aperture of dynamic circular collimators (Iris ${ }^{\mathrm{TM}}$ ).

Silicon detectors based on either $n^{+}-p$ or $p^{+}-n$ junctions would be a sensible choice for monolithic arrays. Their advantages include the potential for manufacturing very small SVs, a stable and near energy-independent response in megavoltage (MV) photon fields, good dose linearity and realtime read-out [7]. They have recently been recommended by Codes of Practice for small-field dosimetry QA [2], [8].

2D monolithic silicon arrays are manufactured on doped wafers, which are then implanted to create pixels (radiation SVs). The substrate of choice has long been a p-type lowresistivity wafer in order to improve the linearity with the dose rate [5].

Unfortunately, these devices are affected by significant radiation damage, resulting in the production of deep level defects, such as interstitial and vacancy defects, and generation-recombination (G-R) centres via interaction of secondary electrons with the detector substrate [9], [10]. G-R centres capture excess minority carriers and facilitate recombination with majority carriers [11]-[13]. Because each defect introduces its own energy level and thus contributes to the overall recombination efficiency, the lifetime $\tau$ of minority charge carriers, i.e. the average time the excess minority carrier needs to recombine, depends on the overall defect structure in the substrate. Other than the relative contribution of each defect energy level, the minority lifetime $\tau$ depends on the injection level and temperature [7]. In the general form, its complex expression is based on Reed-Shockley recombination theory [7]. 
As a first approximation, the variation of defect concentration explains the variation of the minority carrier lifetime, i.e. the average time the excess minority carrier needs to recombine, which for a p-type substrate can be expressed as [5], [14], [15]:

$$
\tau_{\mathrm{e}}=\frac{1}{\sigma_{\mathrm{e}} v_{\mathrm{th}} \mathrm{N}_{\mathrm{t}}} \#(1)
$$

with $\sigma_{\mathrm{e}}$ the cross-section for capture of electrons, $v_{\text {th }}$ their thermal velocity in the lattice at a specific temperature and $\mathrm{N}_{\mathrm{t}}$ the defect concentration in the substrate. As the radiationinduced defects increase with accumulated dose, $\tau_{\mathrm{e}}$ decreases. This is reflected in the minority carrier diffusion length through:

$$
\mathrm{L}_{\mathrm{e}}=\sqrt{\mathrm{D}_{\mathrm{e}} \tau_{\mathrm{e}}} \#(2)
$$

where $\mathrm{D}_{\mathrm{e}}$ is the minority carrier diffusion constant. The sensitivity per unit area of a thick silicon device is directly proportional to its minority carrier diffusion length $L_{e}[5]$ :

$$
\mathrm{S} \propto \mathrm{L}_{\mathrm{e}} \#(3)
$$

Therefore, a decrease in the minority carrier lifetime $\tau_{\mathrm{e}}$ leads to a sensitivity degradation with accumulated dose [5], [15].

Pre-irradiation of the device up to $10 \mathrm{kGy}$, while reducing the sensitivity, allows for its stabilization [16]. This is explained by the saturating behaviour of $\tau$ with accumulated dose [7]. In order to counter the increase of dark current due to pre-irradiation [7], [17], a device can be operated without applying an external bias, i.e. in 'passive' mode. In this case, the depleted region is only a few microns thick, its thickness a function of the built-in potential [18], and the current generated by radiation is controlled by the diffusion current of the excess minority carriers [11].

More recently, it has been shown that it is possible to achieve a constant sensitivity almost independent of the accumulated dose by fixing the SV in two directions: laterally, by using guard-rings, and in depth, by growing onto a highly conductive substrate an epitaxial layer whose thickness is shorter than the $\mathrm{L}_{\mathrm{e}}$ expected in the operative dose range [5], [19]. It was demonstrated that, for an epitaxial device with a thickness of $50 \mu \mathrm{m}$ grown on a Czochralski $(\mathrm{Cz})$ substrate, if:

$$
\mathrm{L}_{\mathrm{e}} \geq \mathrm{W}, \mathrm{r \# (4)}
$$

with $\mathrm{W}$ the epitaxial layer thickness and $r$ the guard ring-SV distance, the active volume $\mathrm{V}$ did not change significantly even at the highest accumulated dose, resulting in a stable sensitivity [5], [19].

The Centre for Medical Radiation Physics (CMRP), University of Wollongong, has designed and characterized two generations of 2D monolithic silicon array devices. The $1^{\text {st }}$ generation (MP512 and Duo [4], [6], [20]) was fabricated on a bulk p-type silicon substrate and featured 512 SVs. In the case of the MP512 the SVs were uniformly distributed on the silicon wafer surface with a $2 \mathrm{~mm}$ pitch, whereas for the Duo they were arranged with $200 \mu \mathrm{m}$ pitch along 2 linear orthogonal arrays.

Whilst the MP512 and the Duo performed excellently under flattened beam (FB) irradiation in terms of OFs, OARs,
PDD distribution, with a small dose per pulse (DPP) dependence [4], [6], [20], their applicability for small-field dosimetry was impaired by the coarse spatial resolution of the MP512 and by the limited spatial characterization of the 2D dose map offered by the Duo.

Thus, a new $2^{\text {nd }}$ generation device 'Octa' has been developed. It was shown that the peculiar layout of the SVs of the Octa has unique potentials for small-field dosimetry, providing a more detailed 2D dose map characterization without sacrificing the necessary spatial resolution. It allows for the simultaneous measurement of $\mathrm{OF}$, cross-plane, in-plane and 2 diagonal OARs for any given radiation field, with submillimetre resolution. [21], [22].

The Octa was produced in two versions, on a bulk and on an epitaxial substrate. We report on their numerical modelling and experimental characterization discussing the effects of resistivity and defects concentration profiles across their largearea monolithic silicon wafers in terms of response linearity with dose, response uniformity and charge-collection efficiency (CCE). Their performance is assessed with respect to small-field dosimetry for medical QA applications.

\section{MATERIALS AND METHODS}

\section{A. The Octa}

The Octa (Figure 1) is a 2D monolithic silicon array detector based on a p-type silicon substrate. Its 512 ionimplanted $\mathrm{n}^{+}$SVs are arranged along 4 intersecting orthogonal linear arrays, oriented 45 degrees with respect to each other. The SVs have all the same area $\left(0.032 \mathrm{~mm}^{2}\right)$ and are of elongated rectangular shape $(40 \mu \mathrm{m} \times 800 \mu \mathrm{m})$, except for the $9 \mathrm{SVs}$ in the central matrix at the intersection of the arrays $(160 \mu \mathrm{m} \times 200 \mu \mathrm{m})$.

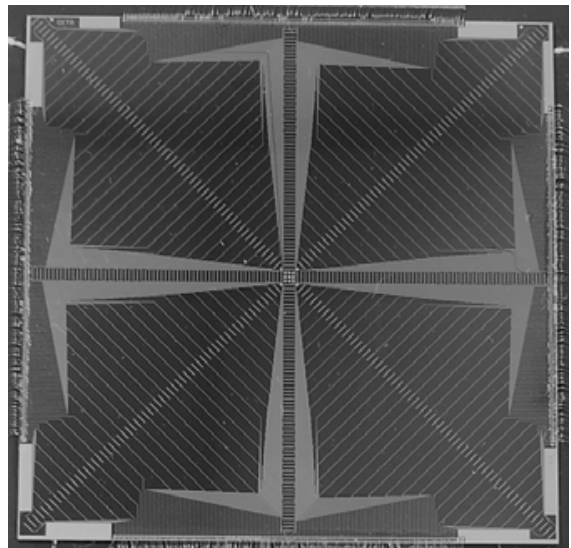

Figure 1. Snapshot of the Octa. The device is a 2D monolithic silicon array detector consisting of 512 diode-SVs operated in passive mode. They are arranged along 4 intersecting orthogonal linear arrays oriented 45 degrees with respect to each other. Each diode has a sensitive area of $0.032 \mathrm{~mm}^{2}$, with a $300 \mu \mathrm{m}$ pitch along the vertical and horizontal arrays and a $430 \mu \mathrm{m}$ pitch along the 2 diagonals.

As a requirement of the SVs ion-implantation planartechnology and for silicon surface passivation, a silicon dioxide $\mathrm{SiO}_{2}$ layer is formed on top of the silicon wafer. The 
layer accumulates positive charges attracting electrons. The accumulation of electrons at the interfaces between the layer and the SVs may short the latter. Non-isolated implants may be detrimental to the $2 \mathrm{D}$ spatial resolution of the device. As a solution, $\mathrm{p}^{+}$stop areas are implanted in between the $\mathrm{n}^{+}$SVs. These re-shapes the electric field of the SVs $n^{+}$-p junctions, cutting into the accumulation layer and preventing the shortening.

The SVs pitch is sub-millimetre, $300 \mu \mathrm{m}$ along the vertical and horizontal arrays and $430 \mu \mathrm{m}$ along the diagonal arrays.

The device has a total area of $38.7 \mathrm{~mm} \times 38.7 \mathrm{~mm}$ and is covered by a $100 \mu \mathrm{m}$ thick layer of epoxy resin to provide a tissue equivalent protection against moisture and accidental damage. Conceived for dose measurements in solid water, it is sandwiched between two Perspex plates, each $5 \mathrm{~mm}$ thick.

The Octa is wire bonded to a $200 \mu \mathrm{m}$ thick printed circuit board (PCB) for connection to a multichannel read-out dataacquisition (DAQ) system, which is based on a commercially available analogue front-end (AFE0064, Texas Instruments), which was described in detail elsewhere [20], [23].

The first version of the Octa was manufactured (SPA-BIT, Kiev, Ukraine) on a $460 \mu \mathrm{m}$ bulk p-type substrate (resistivity $10 \Omega \mathrm{cm}$ ). The silicon wafer was created using a Czochralski process [24]. The bulk Octa sensitivity was stabilized by preirradiation [16] with a Co-60 gamma source at the Gamma Technology Research Irradiator (GATRI) facility at the Australian Nuclear Science and Technology Organisation (ANSTO) in the order of $12 \mathrm{Mrad}$.

The second version of the Octa was manufactured (SPABIT, Kiev, Ukraine) on a $35 \mu \mathrm{m}$ p-type epitaxial [25] layer (resistivity $100 \Omega \mathrm{cm}$ ), grown onto a $525 \mu \mathrm{m}$ thick heavily doped silicon substrate with resistivity $0.001 \Omega \mathrm{cm}$.

The topology in both bulk and epitaxial versions was the same.

\section{B. Radiation damage and electrical characterization}

A current-voltage I-V characterization is a standard test to determine the baseline leakage current and assess the uniformity of the diodes response. In this study, reverse current voltage I-V characteristics measurements were performed using an automatic Semiconductor Measurement Unit (SMU) 237 from Keithley, at a constant laboratory temperature of $24^{\circ} \mathrm{C}$. The diodes reverse bias was investigated in the range from $0 \mathrm{~V}$ to $100 \mathrm{~V}$. The bias was applied to the backside contact.

A capacitance-voltage $(\mathrm{C}-\mathrm{V})$ characterization is a test aimed at determining the device full depletion voltage. In this study, measurements were performed with a bridge capacitance meter Boonton 7200 , at a constant laboratory temperature of $24^{\circ} \mathrm{C}$. The diodes bias was investigated in the range from $0 \mathrm{~V}$ to $20 \mathrm{~V}$.

For both $\mathrm{I}-\mathrm{V}$ and $\mathrm{C}-\mathrm{V}$ characterizations, measurements were carried out for one $\mathrm{SV}$ at a time, randomly located on the silicon wafer. Neighbouring SVs were not grounded during measurements. The effect of this on the magnitude of the collected currents was neglected for the scope of this discussion.

\section{Linearity}

A characterization of the linearity of the bulk Octa response was performed by irradiating the device at $1.5 \mathrm{~cm}$ depth in a water equivalent phantom at $100 \mathrm{~cm}$ surface-to-source distance (SSD) with a $20 \mathrm{~cm}$ side square flat field with a $6 \mathrm{MV}$ flattened beam (FB) delivered by a medical linear accelerator. In these conditions, at $1.5 \mathrm{~cm}$ depth, $1 \mathrm{MU}$ delivered by the accelerator corresponds to $1 \mathrm{cGy}$. The response linearity was investigated in the range of $50 \mathrm{MU}$ to $500 \mathrm{MU}$.

The linearity of the epitaxial Octa response was performed using the same experimental settings, but with the device at $10 \mathrm{~cm}$ depth in the phantom, owing to different availability of solid water slabs at that time.

\section{Uniformity}

Ideally, the response of the Octa 512 diodes would be uniform when the device is irradiated in a flat field. However, this is not the case, owing to unavoidable non-uniformity of the original silicon wafer and possible variations involved in the fabrication processes. A key issue is the presence of defects within the silicon material, intrinsic or due to radiation induced damage. Differences in their local concentration lead to variances in the electric field distribution and charge trapping/recombination process.

Understanding of this change in the electrostatic and charge collection behaviour of the device is especially relevant when operating the device in passive mode.

Other factors affecting a diode response are the parasitic capacitance associated with different length of the connecting leads to each SV and variation in preamplifiers gain in multichannel read-out system, which can vary within $0.1 \%$ to $0.5 \%$ of the dynamic range [26].

The non-uniformity of the integral response can be addressed with an equalization procedure requiring the irradiation of the device with a flat radiation field and then the application of the corresponding equalization factors.

The Octa was irradiated at $10 \mathrm{~cm}$ depth $90 \mathrm{~cm}$ SSD in a water equivalent phantom with a $20 \mathrm{~cm}$ side square flat field with a 6 MV FB delivered by a medical linear accelerator. An equalization factor for each diode was obtained by normalizing each channel response $\mathrm{X}_{\mathrm{i}}$ to the average response of all channels $\langle X\rangle$ to the flat field. The equalization factor was defined as:

$$
\mathrm{F}_{\mathrm{i}}=\frac{\mathrm{X}_{\mathrm{i}}}{\langle\mathrm{X}\rangle} \#(5)
$$

The equalized response $X_{e q, i}$ of each diode was then:

$$
X_{\text {eq,i }}=\frac{X_{i}}{F_{i}} \#(6)
$$

The uniformity $\mathrm{X}_{\%}$ of the 512 diodes response was calculated as:

$$
X_{\%}=\frac{X_{e q}-\langle X\rangle}{\langle X\rangle} \times 100 \#(7)
$$




\section{E. Simulation models of the Octa}

Technology computer-aided design (TCAD) is a simulation tool for semiconductor devices modelling and performance analysis. TCAD simulations were performed using Sentaurus ${ }^{\mathrm{TM}}$ Workbench [27] within the Synopsys ${ }^{\circledR}$ (Synopsys, Inc., Mountain View, CA) framework.

The Sentaurus TCAD software solves the Poisson and carrier continuity equations using finite element methods on a discretised mesh, user-defined and optimized for any given geometry. This mesh-like grid structure of nodes is loaded into the Sentaurus Device (Sdevice) [28] simulation tool.

Depending on the device under investigation and the level of accuracy required, different transport models, each based on a different expression to compute the current densities, can be selected in Sdevice. The drift-diffusion model, which considers the effect of thermal diffusion and the drift caused by the local electric field resulting from applied bias (if any) and electrostatic forces between carriers, was used.

Defects in the substrate reduce charge collection by various generation-recombination processes. Recombination through deep defect levels in the semiconductor energy gap is modelled using the Shockley-Read-Hall (SRH) recombination theory. The SRH lifetimes dependence on doping profiles is modelled in Sdevice through the Scharfetter relation [28].

The Trap model in the Physics section of the Sdevice command file allows for the parametrization of the trapped charge at the interfaces and of the point defects in the substrate, specifying the energy levels, the concentration as a function of the accumulated dose and the cross-section for electrons and holes.

Radiation incident on a semiconductor triggers the generation of electron-hole pairs (ehp). In the Physics section of the Sdevice command file it is possible to model the carrier generation through the Gamma Radiation Model. The user can define a dose rate (rad/s) and the irradiation duration. Alternatively, a Heavy Ion Model can be used. The model is used to represent a minimum ionising particle (MIP) incident on the device. The charge deposited by the particle along a track, or its linear energy transfer (LET) generation density $\left(\mathrm{ehp} / \mathrm{cm}^{3}\right)$, is a user-defined parameter, along with track length, incident location and direction, and lateral distribution. A detailed descriptions of these models can be found in the Sentaurus-Device User Guide and references therein [28].

Using the Sentaurus Structure Editor (SDE) [29], 2D TCAD devices representative of the bulk and epitaxial Octa were created. For both devices, the considered SV was $40 \mu \mathrm{m}$ wide. Other parameters (pitch and number of modelled SVs per single device) were variable in the simulations.

The radiation damage of the pre-irradiated bulk Octa was considered by implementing the Trap model. As reported in the literature, defects generated in a silicon substrate by a Co60 gamma source can be effectively modelled by introducing interstitial $C_{i} O_{i}$ complexes and $V V$ divacancy centres in the substrate, as well as positive trapped charge at the interfaces with and within the silicon dioxide layers [30].

Following recommendations in Aldosari et al. [18] and references therein, a two-level radiation damage model was implemented for the silicon substrate (see Table 1). Following recommendations reported in the same references, a concentration of trapped charges at the $\mathrm{Si}-\mathrm{SiO}_{2}$ interfaces and within the $\mathrm{SiO}_{2}$ layers of $\mathrm{C}=10^{12} \mathrm{~cm}^{-2}$ and $\mathrm{C}=10^{7} \mathrm{~cm}^{-2}$ for the pre-irradiated bulk Octa and for the epitaxial Octa respectively was considered. The concentration saturates between $1.5 \times 10^{12} \mathrm{~cm}^{-2}$ and $3.5 \times 10^{12} \mathrm{~cm}^{-2}$ [31].

The Mobility model was declared in the Physics section of the Sdevice command file to implement a SRH dopingdependent process.

The TCAD devices were validated against experimentally determined $\mathrm{I}-\mathrm{V}$ and $\mathrm{C}-\mathrm{V}$ characteristics, with doping concentrations and profiles tuned to fit the experimental results. Avalanche models available to simulate the breakdown voltage were not considered for the scope of this discussion.

The Heavy Ion Model and the Gamma Radiation Model were used to investigate the CCE in the Octa's SVs as a function of pitch and substrate parameters, at zero bias.

In a first scenario, the MIP simulated with the Heavy Ion Model had a normal incidence on the device with a continuous charge distribution generation of $80 \mathrm{ehp} / \mu \mathrm{m}$. The CCE was defined as:

$$
\operatorname{CCE}(\%)=\frac{Q_{j, x}}{Q_{j, x=0}} \times 100 \#(8)
$$

$\mathrm{Q}_{\mathrm{x}=0}$ is the charge collected by the $\mathrm{SV} j$, taken as the integrated current, when the MIP hits at its centre; $Q_{j, x}$ is the charge collected by the same SV when the MIP hits at a distance $x$ from its centre. The Octa was modelled with $5 \mathrm{SVs}$ and $j$ was the middle SV.

In a second scenario, using the Gamma Radiation Model, the dose rate was of $4.2 \times 10^{4} \mathrm{rad} / \mathrm{s}$ for a $5 \mu$ s duration, representative of a typical medical linac measurement condition. The CCE was defined as:

$$
\operatorname{CCE}(\%)=\frac{Q_{j, 5 p}}{Q_{j, 1 p}} \times 100 \#(9)
$$

$\mathrm{Q}_{\mathrm{j}, 5 \mathrm{p}}$ is the charge collected by the $\mathrm{SV} j$, taken as the integrated current, when the simulated device has $5 \mathrm{SVs}$ and $j$ is the middle one; $Q_{j, 1 p}$ is the charge collected by the same $\mathrm{SV}$, when the simulated device is the same as the previous one, but has only the one SV.

Table 1. Two-level radiation damage model. $\mathrm{D}$ is the dose in water in units of kGy [18].

\begin{tabular}{cclll}
\hline \hline Energy $[\mathrm{eV}]$ & Type of defect & \multicolumn{2}{l}{$\begin{array}{l}\text { Introduction } \\
\text { rate }\left[\mathrm{cm}^{-1}\right]\end{array}$} & \multicolumn{2}{c}{ Cross section $\left[\mathrm{cm}^{-2}\right]$} \\
& & & Electrons & Holes \\
\hline$E_{v}+0.36$ & $C_{i} O_{i}$ donor & 1.826 & 2.5 & 2.5 \\
& & $\times 10^{12} \times \mathrm{D}$ & $\times 10^{-14}$ & $\times 10^{-15}$ \\
$E_{c}-0.42$ & $V V^{(-0)}$ acceptor & 3.040 & 2.0 & 2.0 \\
& & $\times 10^{12} \times \mathrm{D}$ & $\times 10^{-15}$ & $\times 10^{-14}$ \\
\hline \hline
\end{tabular}

\section{F. Clinical application}

As a clinical application, we considered the measurement of out of axis ratios (OARs). Experimental measurements 
described in this study were performed at the Sir Charles Gairdner Hospital (SCGH), Nedlands, WA, Australia.

The Octa was irradiated with a $6 \mathrm{MV}$ flattening filter free (FFF) beam using an Accuray CyberKnife ${ }^{\circledR}$ M6 linear accelerator (Accuray, Palo Alto, CA). Radiation field sizes were defined using a dynamic circular collimator called Iris ${ }^{\mathrm{TM}}$ (Accuray, Palo Alto, CA).

OARs were measured by the Octa on top of a $10 \mathrm{~cm}$ solid water slab for backscattering purposes, at $1.5 \mathrm{~cm}$ depth and $80 \mathrm{~cm}$ source-to-detector distance (SDD). IBA solid water slabs type RW3 were used. The initial experimental setup is shown in Figure 2, with the Octa set on the treatment couch.

Prior to the measurements, the Octa was aligned with respect to the treatment machine central axis (CAX) by maximizing the response of its central SV using the smallest available field size ( $5 \mathrm{~mm}$ diameter). Once aligned, for any given field size, OARs (in-line, cross-line and 2 diagonals) were measured simultaneously. The readings of the Octa at each field size were taken as the reading of each channel averaged over 3 repetitions of the same measure followed by normalisation of the response of each channel to the median response of the SVs within $0.5 \mathrm{~mm}$ of the CAX. For each profile, estimates were made of the full width at halfmaximum (FWHM) and the penumbra width (taken as the distance between the $80 \%$ and the $20 \%$ isodose levels).

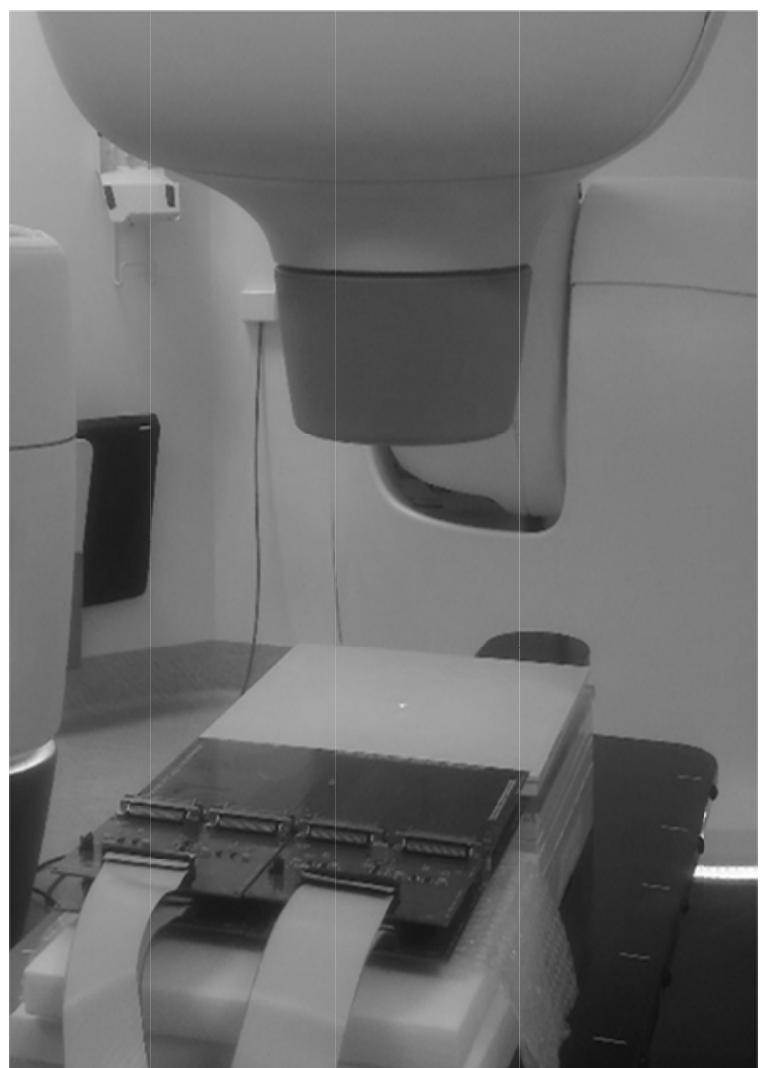

Figure 2. Experimental setup at the SCGH. The Octa detector was set on the treatment couch on top of $10 \mathrm{~cm}$ solid water for backscattering purposes. Solid water slabs were then added on top of the detector to reach the water equivalent depth required for each measurement.

Measurements by the Octa were compared with those made using a PTW SRS diode 60018 mounted parallel to the axis in an IBA 3D water-phantom. The diode was oriented vertically, measuring at the effective point of measurement of $1.3 \mathrm{~mm}$ from top surface. Its readings were corrected using the correction factors by Francescon et al. [32].

\section{RESULTS AND DISCUSSION}

\section{A. Radiation damage and electrical characterization}

The simulated space-charge distribution (Figure 3) for one of the Octa $\mathrm{n}^{+}$electrodes (SV), along with its $\mathrm{p}^{+}$stop, shows that the depleted region is stretched outside the limits of the junction area due to the presence of charges in the silicon oxide layer (dark brown in the picture), however do not spread inside of the $\mathrm{p}^{+}$stop area .

The depleted region depth for the Octa epitaxial under zero bias was estimated to be approximately between $1 \mu \mathrm{m}$ and $2 \mu \mathrm{m}$, which is consistent with those reported for dosimeters based on p-n junctions operated without any external bias [5] along with values simulated for a similar epitaxial device presented by Aldosari et al, 2013 [18].

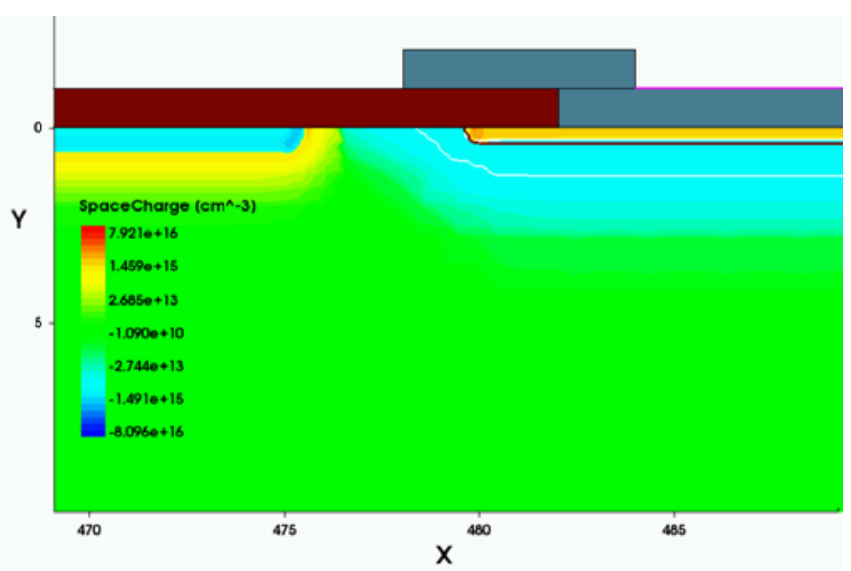

Figure 3. Simulated representation of the space-charge distribution for an epitaxial Octa. The depletion region (white line) is stretched outside the limits of the $\mathrm{n}^{+}-\mathrm{p}$ junction (brown line) due to the presence of charges in the $\mathrm{SiO}_{2}$ layer (brown area). Grey areas represent the aluminum contact of the $\mathrm{n}^{+}$electrode (SV). The $\mathrm{p}^{+}$ stop area is visible on the left. Distances are in microns.

The leakage current $\mathrm{I}_{\text {leak }}$ is related to the applied voltage $\mathrm{V}$ across the device through [30]:

$$
\mathrm{I}_{\text {leak }} \propto \mathrm{W} \propto \sqrt{\mathrm{V}} \text { for } \mathrm{V} \leq \mathrm{V}_{\mathrm{dep}} \#(10)
$$

with $\mathrm{W}$ the thickness of the depleted region.

The fact that the depletion is not only under the SVs but is spread laterally (because of their small size) explains the deviation of the experimental measurements from the ideal behaviour. 

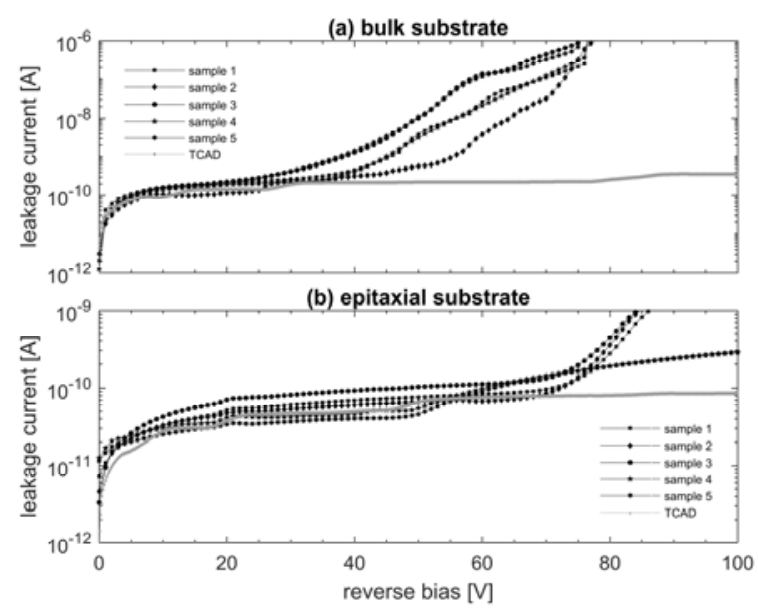

Figure 4. Family of experimental I-V characteristics from a few sample diodes (SVs) of the (a) bulk and (b) epitaxial Octa, along with the simulated characteristic.

The leakage current is also proportional to [30]:

$$
I_{\text {leak }} \propto \frac{W}{\tau_{\mathrm{g}}} \#(11)
$$

with $\tau_{\mathrm{g}}$ the generation lifetime, i.e. the time it takes on average to generate an electron-hole pair, a parameter which is inversely proportional to the impurity density and the capture cross-section for electrons and holes but is in general not equal to the recombination lifetime [14].

Therefore, variations in the values of the I-V characteristics among diodes (SVs) on the same device are, in part, explained by their specific position within the silicon wafer and heterogeneous distribution of defects and doping concentration.

The variation of $I_{\text {leak }}$ as a function of accumulated dose, which would reflect the increasing concentration of radiationinduced defects in the silicon and $\mathrm{Si}-\mathrm{SiO}_{2}$ interfaces, has previously been characterized for similar bulk and epitaxial substrates elsewhere [18], [33].

When considering the I-V characterization of the TCAD model, an area factor was introduced to normalize the $\mathrm{I}_{\text {leak }}$ so that the model had the same volume of the experimental device.

Simulated I-V characteristics of a single SV, in a device modelled with $5 \mathrm{SVs}$, were found to follow those of an ideal junction and fit within the range of the experimental measurements, for both the thick and epitaxial Octa (Figure 4).

The capacitance $C$ of the junction is given by [15]:

$$
\mathrm{C}=\frac{\varepsilon_{0} \varepsilon_{\mathrm{Si}}}{\mathrm{W}}=\sqrt{\frac{\mathrm{q} \varepsilon_{0} \varepsilon_{\mathrm{Si}} \mathrm{N}_{\mathrm{eff}}}{2\left(\mathrm{~V}_{\mathrm{bl}}-\mathrm{V}\right)}} \#(12)
$$

with $\mathrm{W}$ the width of the depleted region, $\varepsilon_{0}$ is the permittivity of free space, $\varepsilon_{\mathrm{Si}}$ is the dielectric constant of silicon, $V_{\mathrm{bl}}$ is the junction built-in potential, $\mathrm{V}$ is the applied reverse bias, $\mathrm{N}_{\text {eff }}$ the doping concentration. By increasing the applied bias across the device, the capacitance $C$ decreases as [30]:

$$
\mathrm{C} \propto \frac{1}{\sqrt{\mathrm{V}}} \#(13)
$$

up to when full depletion is reached, which was not reached in our study due to the low resistivity values of the considered silicon. $V_{\mathrm{depl}}$ is the applied reverse bias, at which the entire detector volume is depleted of free charge carriers. At that point, applying any higher voltages would not change the depletion depth, hence the measured capacitance.

For small SVs, though, lateral depletion is very pronounced, and capacitance decreases even after full depletion.
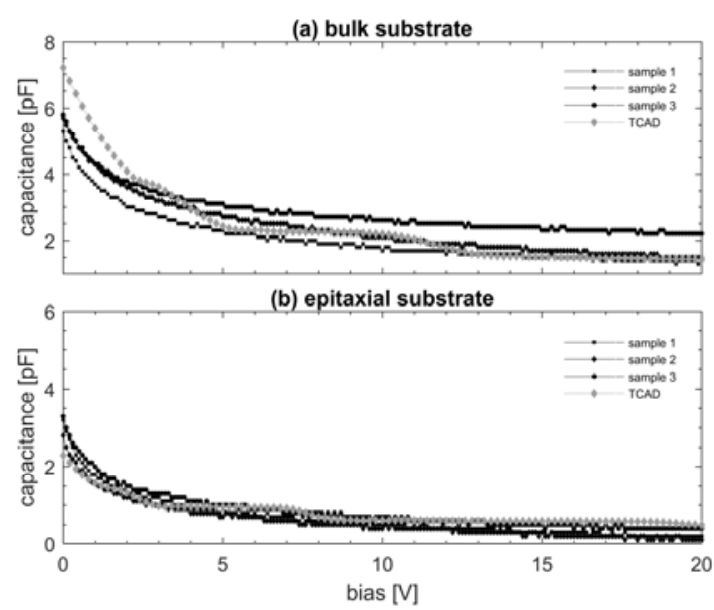

Figure 5. Family of experimental $\mathrm{C}-\mathrm{V}$ characteristics from a few sample diodes (SVs) of the (a) bulk and (b) epitaxial Octa, along with the simulated characteristic.

When considering the $\mathrm{C}-\mathrm{V}$ characterization of the TCAD model, an area factor was introduced to normalize the capacitance so that the model had the same volume of the experimental device.

Simulated C-V characteristics of a single SV, in a device modelled with $5 \mathrm{SVs}$, were found to fit within the range of the experimental measurements, for both the thick and epitaxial Octa (Figure 5).

The device packaging, though, which is not accounted for in the simulations, results in an increase in the real device total capacitance. This discrepancy value was subtracted from the experimental characteristics for the entire range of bias measured.

The simulated devices were found to reproduce experimental $\mathrm{I}-\mathrm{V}$ and $\mathrm{C}-\mathrm{V}$ characterizations when modelled with a resistivity of $4 \Omega \mathrm{cm}$ and $40 \Omega \mathrm{cm}$ for the bulk and epitaxial Octa respectively.

\section{B. Linearity}

In terms of response linearity with delivered dose, the bulk Octa results are, as expected, consistent with those of similar bulk devices previously characterized, such as the MP512 [34] and the Duo [33]. Figure 6 shows that the epitaxial Octa demonstrated an equally good linear response. In both figures, 
error bars, calculated as 2 standard deviations, did not exceed the symbol size.
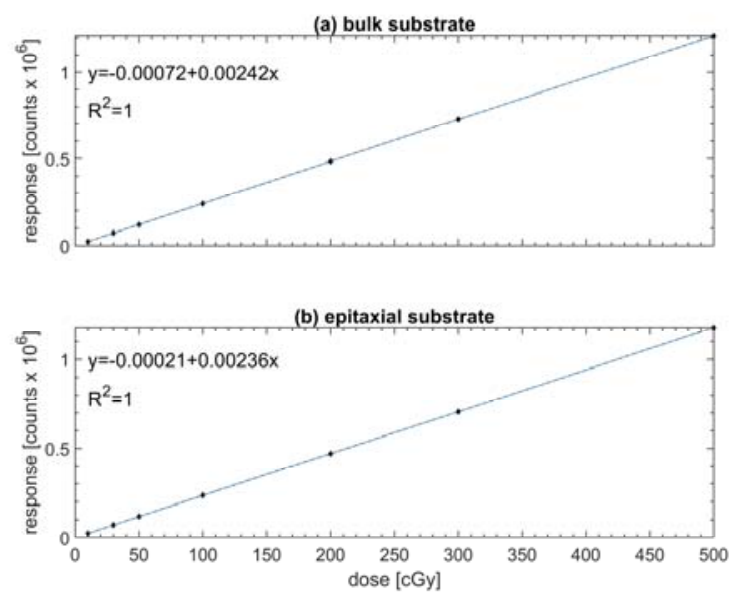

Figure 6. Linearity response of (a) bulk and (b) epitaxial Octa. The regression coefficient $R^{2}$ is 1 in both cases.

\section{Uniformity}

When irradiated in a flat field, the epitaxial Octa demonstrated a more uniform response than its bulk counterpart (Figure 7, Figure 8).

Since the same data acquisition system was used for both detectors, this result is mainly explained by the much better uniformity and quality of the epitaxial silicon wafer in terms of resistivity and recombination properties.

For the epitaxial device, the diodes along the diagonal arrays were found to be slightly more sensitive than those along the vertical and horizontal arrays (Figure 7). This overresponse, due to the SVs larger collection area allowed by their greater pitch, is addressed by applying the equalization factors.

Based on previous radiation damage studies [33], it is estimated that the sensitivity of the bulk Octa has been reduced by approximately $55 \%$ as a consequence of its preirradiation. Conversely, the sensitivity of the epitaxial Octa, which was not pre-irradiated, could be expected to increase, albeit slightly, with future exposure to irradiation as a consequence of continuous clinical testing [18].
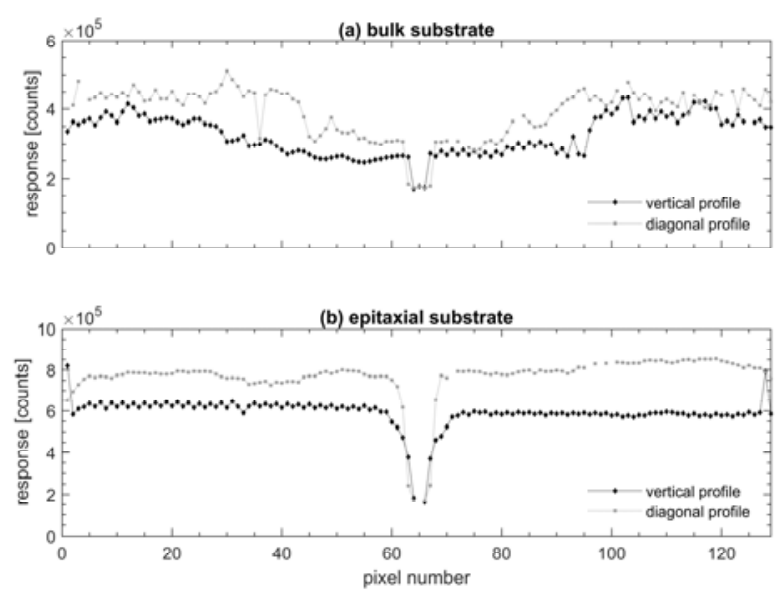

Figure 7. Response to a flat field, with no equalization applied, of the diodes along the vertical $(300 \mu \mathrm{m}$ pitch) and diagonal $(430 \mu \mathrm{m}$ pitch) arrays of the (a) bulk and (b) epitaxial Octa.
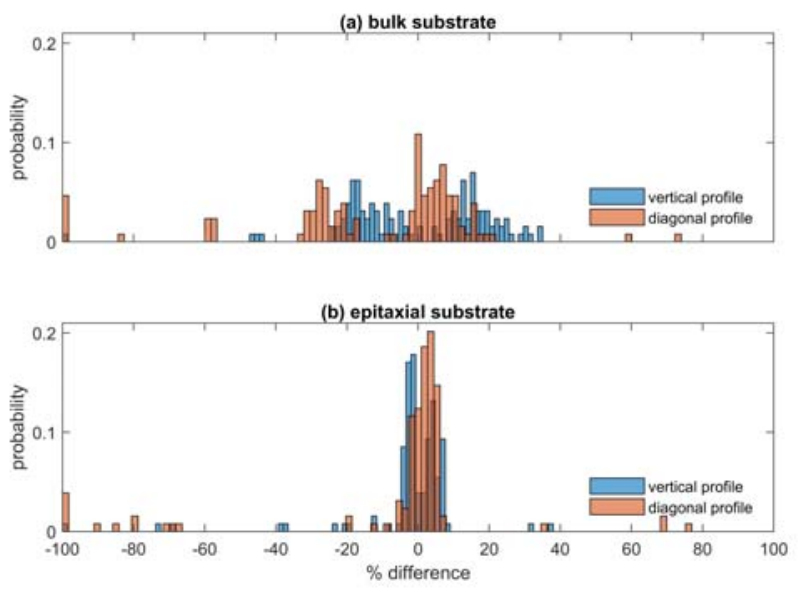

Figure 8. Statistical distribution of the SVs response to a flat field, with no equalization applied, for the (a) bulk and (b) epitaxial Octa.

\section{Charge collection efficiency and spatial resolution}

According to TCAD simulations performed using the Heavy Ion Model (Figure 9), for a bulk Octa CCE becomes negligible $(<0.5 \%)$ approximately at $60 \mu \mathrm{m}$ from the SV centre.

For an epitaxial Octa, CCE was $2.75 \%$ at a distance of $300 \mu \mathrm{m}$ from the SV centre, for the $300 \mu \mathrm{m}$ pitch, and $<1 \%$ at a distance of $430 \mu \mathrm{m}$ for the $430 \mu \mathrm{m}$ pitch.

The difference in CCE between the Octas is mainly explained by the $\tau_{\mathrm{e}}$ of the epitaxial substrate being greater than that for the pre-irradiated bulk substrate, allowing the p-n junction to collect charge over a greater lateral distance with respect to the SV centre.

By considering the distance at which the CCE is $50 \%$, the $430 \mu \mathrm{m}$-epitaxial configuration could be used to estimate that the $\mathrm{L}_{\mathrm{e}}$ for this substrate is approximately $90 \mu \mathrm{m}$. It is proposed that for the pre-irradiated bulk device $\mathrm{L}_{\mathrm{e}}$ is between $20 \mu \mathrm{m}$ and $40 \mu \mathrm{m}$. 

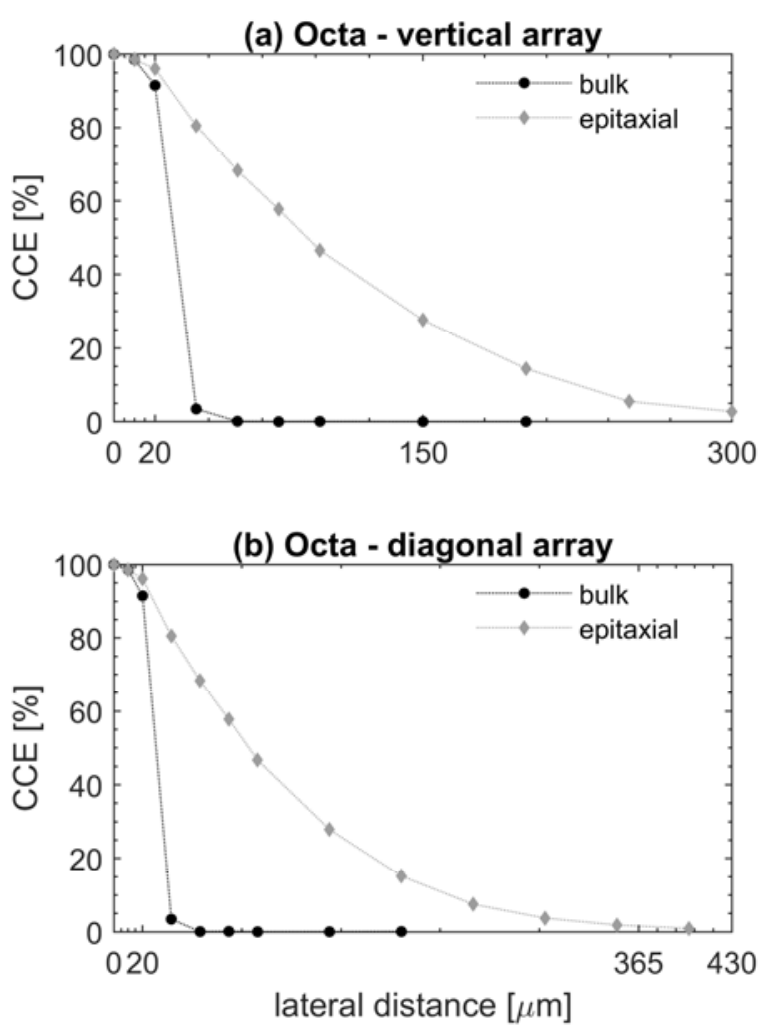

Figure 9. Simulated CCE as a function of the lateral distance from the SV center, for a SV (a) in a $300 \mu \mathrm{m}$ pitch configuration and (b) in a $430 \mu \mathrm{m}$ pitch configuration, for the pre-irradiated bulk Octa and for the epitaxial Octa.

According to TCAD simulations performed using the Gamma Radiation Model, CCE dependence on the SVs' pitch is negligible for the pre-irradiated bulk Octa (Figure 10), whereas it is appreciable for the epitaxial Octa (Figure 11). In this case, the simulated CCE was $50.24 \%$ for a $200 \mu \mathrm{m}$ pitch, $68.19 \%$ for a $300 \mu \mathrm{m}$ and $83.34 \%$ for a $430 \mu \mathrm{m}$ pitch.

The SVs of the epitaxial Octa collect more charge when in a $430 \mu \mathrm{m}$ pitch configuration as compared to a $300 \mu \mathrm{m}$ pitch configuration. Remarkably, TCAD simulations show 18\% increase (Figure 11) which is in close agreement with the findings of experimental measurements which showed on average 24\% increase (Figure 7).

A CCE different from $100 \%$, though, does not mean that the device spatial resolution is affected. Its deterioration would depend on a non-linear charge sharing between neighbouring SVs due to the presence of dose gradients. Experimental characterizations of small beam profiles on MV linear medical accelerators performed by the epitaxial Octa indicates that this is not the case. Both a $300 \mu \mathrm{m}$ pitch configuration and a $430 \mu \mathrm{m}$ pitch configuration were shown to be suitable for high spatial resolution dose mapping [21], [22].

The CCE was also found to depend on the epitaxial layer resistivity (Figure 12), with a saturating behaviour below $0.5 \Omega \mathrm{cm}$, in the case of the $300 \mu \mathrm{m}$ pitch configuration. Decreasing the silicon resistivity would improve the SVs $\mathrm{CCE}$, at the cost of decreased device sensitivity. Defining a minimum value of acceptable resistivity for the silicon on which the Octa is based, however, would require a complex theoretical and experimental evaluation on the impact this would have on the whole system composed of radiation detector proper and read-out electronics. This assessment was beyond the scope of this work.

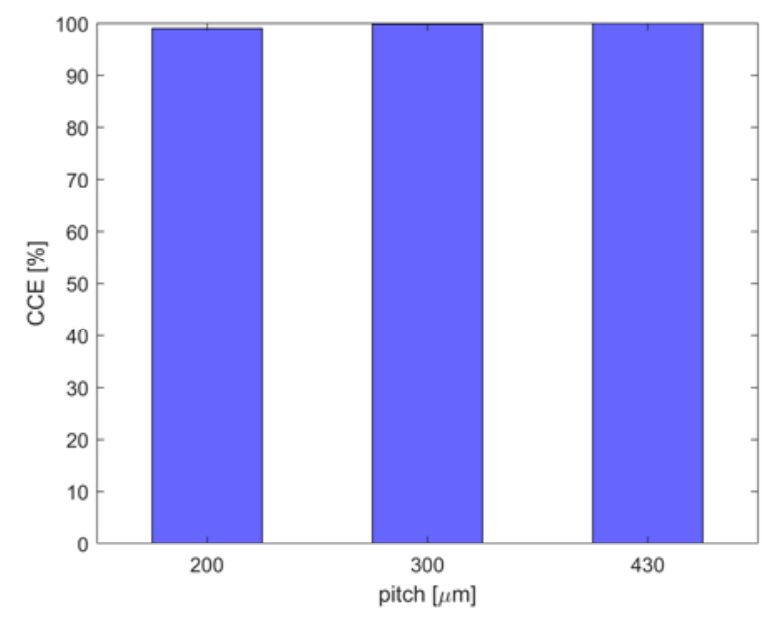

Figure 10. Simulated CCE as a function of the SVs' pitch for the preirradiated bulk Octa, in the case of a resistivity of $4 \Omega \mathrm{cm}$.

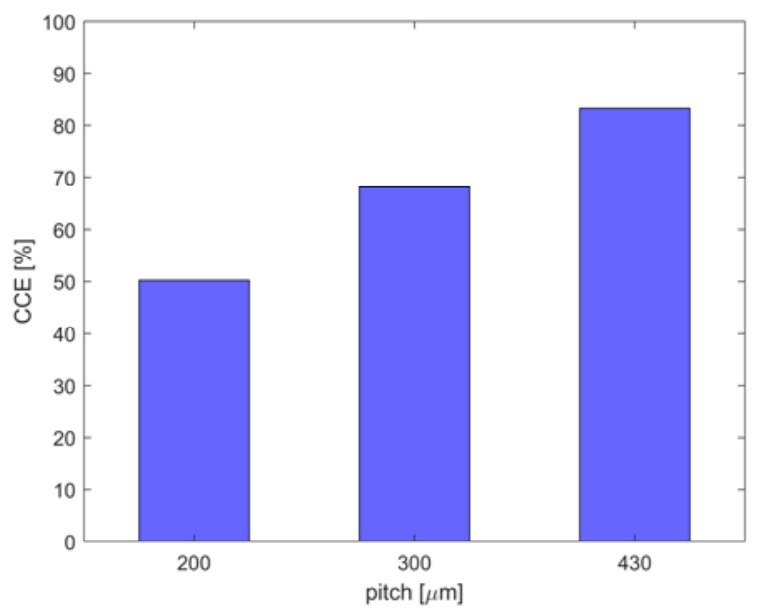

Figure 11. Simulated CCE as a function of the SVs' pitch for the epitaxial Octa, in the case of an epitaxial layer resistivity of $40 \Omega \mathrm{cm}$. 


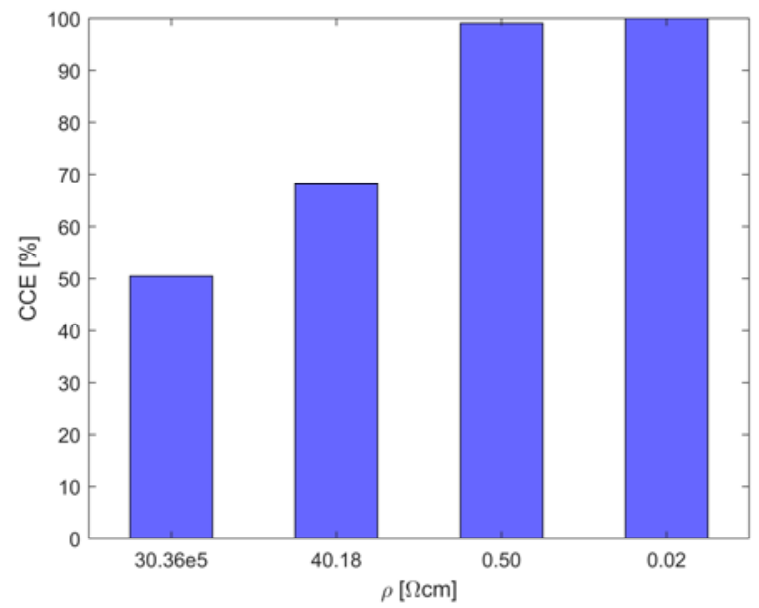

Figure 12. Simulated CCE as a function of the epitaxial layer doping for the $300 \mu \mathrm{m}$ pitch configuration.

\section{E. Clinical application}

A reliable sub-millimetre spatial-resolution of the $2 \mathrm{D}$ dose map in terms of FWHM and penumbra width was demonstrated for both Octas, with results consistent with respect to SRS diode measurements (Table 2).

The in-line profiles for a $3 \mathrm{~cm}$ side circular field measured by the bulk and epitaxial Octas are shown in Figure 13 a) and b) respectively. The full study of the epitaxial Octa as a QA tool for the CyberKnife ${ }^{\circledR}$ linear accelerator is reported elsewhere [22].

Typically, for any given dose measurement, equalization factors from a flat-field acquisition using the same beam quality and the same linear accelerator would have to be applied.

When using the Octa for CyberKnife ${ }^{\circledR}$ QA applications, though, this would be impossible, as the linac is operated only in flattening filter free (FFF) modality and cannot produce flat fields. In this case, a different linac with flattened beam (FB) modality would need to be used for the flat-field acquisition followed by equalization. Critically, the two linacs would be operating at different instantaneous dose rates.

Other than the obvious technical convenience, the reason for using the same linac was to avoid issues arising from the $\tau_{\mathrm{e}}$ dependence on instantaneous the dose rate [11], [12], which affects the sensitivity of the silicon device. But $\tau_{\mathrm{e}}$ is mainly a function of the defects in the substrate, which have been reported in the literature to be arranged in concentric rings across a bulk silicon wafer [15], as a consequence of the manufacturing process. A variation in the local defects concentration results in a sensitivity variation, as a function of the instantaneous dose rate, across the wafer. In other words, recombination properties may be affected by non-uniformities (Figure 7) in a bulk wafer when using different dose rates. In the case presented, the device irradiation was performed with different dose rates for the flat field and for the field to be equalized, and a ring-shaped non-uniformity resulted in the equalized dose profiles for the bulk device (Figure 13). The over-response in a ring of diameter approximately $2 \mathrm{~cm}$ is apparent in the figure and relates the non-uniformities in (Figure 7).

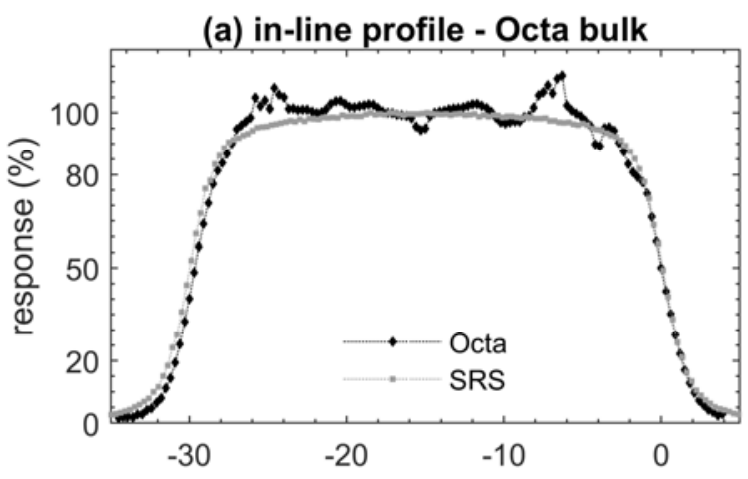

(b) in-line profile - Octa epitaxial

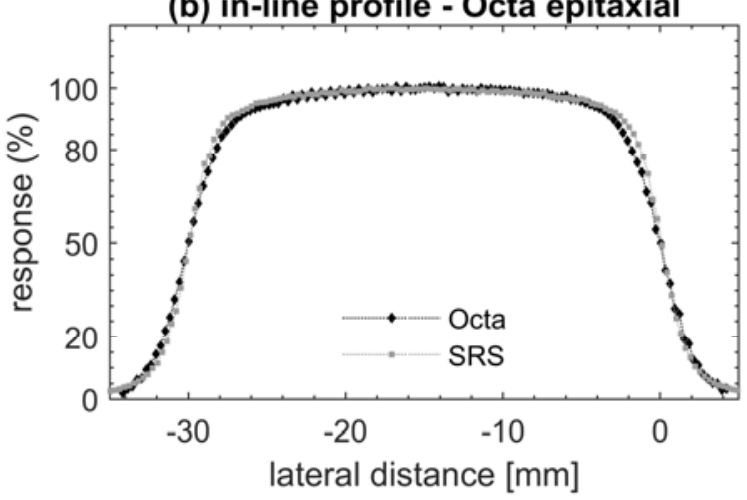

Figure 13. In-line profiles measured by the Octa (a) bulk and (b) an epitaxial substrate. Profiles are for a radiation field collimated with the variable aperture Iris collimator mounted on a CyberKnife ${ }^{\circledR}$ M6. A $6 \mathrm{MV}$ FFF beam quality was used. Data is benchmarked with measurements by a SRS diode and aligned to the $50 \%$ response.

Table 2. FWHM and penumbra width as measured by the Octas. Differences are with respect to measurements performed by an SRS diode in the same experimental settings.

\begin{tabular}{lcccc}
\hline \hline & $\begin{array}{c}\text { FWHM } \\
{[\mathrm{cm}]}\end{array}$ & $\begin{array}{c}\text { Penumbra } \\
{[\mathrm{cm}]}\end{array}$ & $\begin{array}{c}\Delta \text { FWHM } \\
{[\%]}\end{array}$ & $\begin{array}{c}\Delta \text { Penumbra } \\
{[\mathrm{cm}]}\end{array}$ \\
\hline $\begin{array}{l}\text { Octa } \\
\text { (bulk) }\end{array}$ & 2.96 & 0.30 & -0.7 & 0.04 \\
$\begin{array}{l}\text { Octa } \\
\text { (epitaxial) }\end{array}$ & 3.00 & 0.33 & 0.7 & 0.07 \\
SRS diode & 2.98 & 0.26 & - & - \\
\hline \hline
\end{tabular}

On the other hand, for the epitaxial Octa, a much more homogenous distribution of concentration across the epitaxial layer and fewer intrinsic defects, owing to an improved manufacturing process, grants a much more uniform response (Figure 7). Therefore, the equalized inline dose profile in Figure 13 measured by the epitaxial Octa is as smooth as that measured by the SRS diode. 


\section{CONCLUSIONS}

The Octa, a 2D monolithic silicon array detector dedicated to small-field dosimetry, was produced in two samples, on a bulk and on an epitaxial substrate. Their performance was investigated by experimental measurements and TCAD based numerical simulations in terms of response linearity with dose, response uniformity and charge-collection efficiency (CCE). Results were compared and discussed with respect to smallfield dosimetry for medical quality assurance (QA) applications.

TCAD simulations of a minimum ionizing particle (MIP) through the device using the Heavy Ion Model were demonstrated to be a good tool for characterization of the charge collection efficiency (CCE) of a monolithic array detector. The simulated CCE distribution could be used to optimize the pitch and the SVs' layout across the silicon wafer based on the estimated minority carrier diffusion length.

We are not aware of any other study in the literature of the CCE characterization for a monolithic array detector using the Gamma Radiation Model. This was shown to be an invaluable tool for investigating how the detector performance is affected by parameters such the SVs pitch, the silicon resistivity and traps concentration. This methodology provides a means of optimising future devices prior to fabrication.

For a bulk pre-irradiated device, with a sufficiently small $\mathrm{L}_{\mathrm{e}}$, the SVs pitch does not affect their CCE, owing to the charge collection being confined to the geometrical size of the SVs themselves.

For an epitaxial device, if $\mathrm{L}_{\mathrm{e}} \geq \mathrm{W}, \mathrm{r}$, radiation hardness is preserved, and the device does not require pre-irradiation. However, with a comparably higher $\mathrm{L}_{\mathrm{e}}$, the SVs pitch affects their CCE. The charge collection is less confined to the SV and there is a significant lateral diffusion of charge. This could be in principle be addressed by decreasing the silicon resistivity, at the cost of a reduced sensitivity. Nonetheless, even in the presence of a sub-optimal CCE, the detector nominal spatial resolution is expected to be preserved, as supported by previous experimental clinical measurements of the $2 \mathrm{D}$ dose map.

Experimentally, both Octas showed good linearity with dose and a non-uniform response across the whole arrays that could easily be corrected for by applying an equalization procedure. Unfortunately, in the case of contemporary radiotherapy applications with flattening filter free (FFF) beam irradiations, this was demonstrated to be a workable solution only in the case of a device manufactured with a uniform profile in terms of resistivity and recombination properties, i.e. for the epitaxial Octa.

A future investigation will assess the response of the Octas in terms of instantaneous dose rate and angular dependence.

\section{REFERENCES}

[1] M. L. Taylor, T. Kron, and R. D. Franich, "A contemporary review of stereotactic radiotherapy: inherent dosimetric complexities and the potential for detriment," Acta Oncol. (Madr)., vol. 50, no. 4, pp. 483-508, 2011.

[2] ICRU, "ICRU Report 91 - Prescribing, Recording, and Reporting of Stereotactic Treatments with Small Photon Beams," 91, 2017.

[3] F. Bisello, D. Menichelli, M. Scaringella, C. Talamonti, M. Zani, M. Bucciolini, and M. Bruzzi, "Development of silicon monolithic arrays for dosimetry in external beam radiotherapy," Nucl. Instruments Methods Phys. Res. Sect. A Accel. Spectrometers, Detect. Assoc. Equip., vol. 796, pp. 85-88, 2015.

[4] A. H. Aldosari, M. Petasecca, A. Espinoza, M. K. Newall, I. Fuduli, C. S. Porumb, S. AlShaikh, Z. A. Alrowaili, M. R. Weaver, P. Metcalfe, M. G. Carolan, M. L. F. Lerch, V. Perevertaylo, and A. B. Rosenfeld, "A two dimensional silicon detectors array for quality assurance in stereotactic radiotherapy: MagicPlate512," Med. Phys., vol. 41, no. 9, 2014.

[5] M. Bruzzi, "Novel silicon devices for radiation therapy monitoring," Nucl. Instruments Methods Phys. Res. Sect. A Accel. Spectrometers, Detect. Assoc. Equip., vol. 809, pp. 105-112, 2016.

[6] K. Al Shukaili, M. Petasecca, M. K. Newall, A. Espinoza, V. Perevertaylo, S. Corde, M. L. F. Lerch, and A. B. Rosenfeld, "A 2D silicon detector array for quality assurance in small field dosimetry: DUO," Med. Phys., vol. 44, no. 2, pp. 628-636, 2017.

[7] A. B. Rosenfeld, "Electronic dosimetry in radiation therapy," Radiat. Meas., vol. 41, pp. S134-S153, 2007.

[8] IAEA, "Technical Reports Series No.483 - Dosimetry of Small Static Fields Used in External Beam Radiotherapy," 2017.

[9] R. Wunstorf, H. Feick, E. Fretwurst, G. Lindstrom, G. Lutz, C. Osius, R. Richter, T. Rohe, A. Rolf, and P. Schlichtharle, "Damage-induced surface effects in silicon detectors," Nucl. Instruments Methods Phys. Res. Sect. A Accel. Spectrometers, Detect. Assoc. Equip., vol. 377, no. 2-3, pp. 290-297, 1996.

[10] M. Bruzzi, "Radiation damage in silicon detectors for high-energy physics experiments," IEEE Trans. Nucl. Sci., vol. 48, no. 4, pp. 960-971, 2001.

[11] J. Shi, W. E. Simon, and T. C. Zhu, "Modeling the instantaneous dose rate dependence of radiation diode detectors," Med. Phys., vol. 30, no. 9, 2003.

A. S. Saini and T. C. Zhu, "Dose rate and SDD dependence of commercially available diode detectors," Med. Phys., vol. 31, no. 4, pp. 914-924, 2004.

[13] P. A. Jursinic, "Dependence of diode sensitivity on the pulse rate of delivered radiation," Med. Phys., vol. 40, no. 2, 2013.

[14] D. K. Schroder, "Carrier Lifetimes in Silicon," IEEE Trans. Electron Devices, vol. 44, no. 1, pp. 160-170, 
1997.

[15] S. M. Sze and K. K. Ng, Physics of Semiconductor Devices, 3rd ed. John Wiley \& Sons, 2007.

[16] G. Rikner and E. Grusell, "General specifications for semiconductors for use in radiation dosimetry," Phys. Med. Biol., vol. 32, no. 9, 1987.

[17] M. Moll, E. Fretwurst, M. Kuhnke, and G. Lindström, "Relation between microscopic defects and macroscopic changes in silicon detector properties after hadron irradiation," Nucl. Instruments Methods Phys. Res. Sect. B Beam Interact. with Mater. Atoms, vol. 186, no. 1-4, pp. 100-110, 2002.

[18] A. H. Aldosari, A. Espinoza, D. Robinson, I. Fuduli, C. S. Porumb, S. AlShaikh, M. G. Carolan, M. L. F. Lerch, V. Perevertaylo, A. B. Rosenfeld, and M. Petasecca, "Characterization of an innovative p-type epitaxial diode for dosimetry in modern external beam radiotherapy," IEEE Trans. Nucl. Sci., vol. 60, no. 6, pp. 4705-4712, 2013.

[19] M. Bruzzi, M. Bucciolini, M. Casati, D. Menichelli, C. Talamonti, C. Piemonte, and B. G. Svensson, "Epitaxial silicon devices for dosimetry applications," Appl. Phys. Lett., vol. 90, no. 17, pp. 17-19, 2007.

[20] M. Petasecca, M. K. Newall, J. T. Booth, M. Duncan, A. H. Aldosari, I. Fuduli, A. Espinoza, C. S. Porumb, S. Guatelli, P. Metcalfe, E. Colvill, D. Cammarano, M. G. Carolan, B. M. Oborn, M. L. F. Lerch, V. Perevertaylo, P. J. Keall, and A. B. Rosenfeld, "MagicPlate-512: A 2D silicon detector array for quality assurance of stereotactic motion adaptive radiotherapy," Med. Phys., vol. 42, no. 6, pp. 29923004, 2015.

[21] G. Biasi, M. Petasecca, S. Guatelli, N. Hardcastle, M. G. Carolan, V. Perevertaylo, T. Kron, and A. B. Rosenfeld, "A novel high-resolution 2D silicon array detector for small field dosimetry with FFF photon beams," Phys. Medica, vol. 45, pp. 117-126, 2018.

[22] G. Biasi, M. Petasecca, S. Guatelli, M. A. Ebert, G. Grogan, B. Hug, J. Lane, V. Perevertaylo, T. Kron, and A. B. Rosenfeld, "CyberKnife fixed cone and Iris defined small radiation fields: assessment with a highresolution solid-state detector array," J. Appl. Clin. Med. Phys., pp. 1-11, 2018.

[23] I. Fuduli, M. K. Newall, A. Espinoza, C. S. Porumb, M. G. Carolan, M. L. F. Lerch, P. Metcalfe, A. B. Rosenfeld, and M. Petasecca, "Multichannel Data Acquisition System comparison for Quality Assurance in external beam radiation therapy," Radiat. Meas., vol. 71, pp. 338-341, 2014.

[24] J. Czochralski, "Ein neues Verfahren zur Messung der Kristallisations- geschwindigkeit der Metalle," Z. Phys. Chem., vol. 92, pp. 219-221, 1918.

[25] S. M. Sze and K. K. Ng, Semiconductor devices: Physics and Technology, 3rd ed. John Wiley \& Sons, 2006.

[26] Texas Instrument, "64 Channel Analog Front End for Digital X-Ray Detector AFE0064,” no. September. 2009.

[27] Synopsys, "Sentaurus Workbench User Guide." 2013.

[28] Synopsys, "Sentaurus Device User Guide." 2013.
[29] Synopsys, "Sentaurus Structure Editor User Guide." 2006.

[30] M. Moll, "Radiation Damage in Silicon Detectors," PhD Thesis, Universität Hamburg, 1999.

[31] J. Schwandt, E. Fretwurst, E. Garutti, R. Klanner, and I. Kopsalis, "Surface effects in segmented silicon sensors," Nucl. Instruments Methods Phys. Res. Sect. A Accel. Spectrometers, Detect. Assoc. Equip., vol. 845, pp. 159-163, 2017.

[32] P. Francescon, W. Kilby, N. Satariano, and S. Cora, "Monte Carlo simulated correction factors for machine specific reference field dose calibration and output factor measurement using fixed and iris collimators on the CyberKnife system," Phys. Med. Biol., vol. 57, no. 12, pp. 3741-3758, 2012.

[33] C. S. Porumb, A. H. Aldosari, I. Fuduli, D. L. Cutajar, M. K. Newall, P. Metcalfe, M. G. Carolan, M. L. F. Lerch, V. Perevertaylo, A. B. Rosenfeld, and M. Petasecca, "Characterisation of Silicon Diode Arrays for Dosimetry in External Beam Radiation Therapy," IEEE Trans. Nucl. Sci., vol. 63, no. 3, pp. 1808-1817, 2016.

[34] C. S. Porumb, "Development of a multi-detector quality assurance instrument for intensity modulated radiotherapy," $\mathrm{PhD}$ Thesis, University of Wollongong, 2016. 